\title{
The role of imaging in 2019 novel coronavirus pneumonia (COVID-19)
}

\author{
Wenjing Yang ${ }^{1,2,3} \cdot$ Arlene Sirajuddin $^{4} \cdot$ Xiaochun Zhang $^{3} \cdot$ Guanshu Liu $^{5} \cdot$ Zhongzhao Teng $^{6,7} \cdot$ Shihua Zhao ${ }^{1}$. \\ Minjie Lu ${ }^{1,4,8}$
}

Received: 10 March 2020 / Revised: 14 March 2020 / Accepted: 20 March 2020 / Published online: 15 April 2020

(C) European Society of Radiology 2020

\begin{abstract}
Almost the entire world, not only China, is currently experiencing the outbreak of a novel coronavirus that causes respiratory disease, severe pneumonia, and even death. The outbreak began in Wuhan, China, in December of 2019 and is currently still ongoing. This novel coronavirus is highly contagious and has resulted in a continuously increasing number of infections and deaths that have already surpassed the SARS-CoV outbreak that occurred in China between 2002 and 2003. It is now officially a pandemic, announced by WHO on the 11th of March. Currently, the 2019 novel coronavirus (SARS-CoV-2) can be identified by virus isolation or viral nucleic acid detection; however, false negatives associated with the nucleic acid detection provide a clinical challenge and thus make the imaging examination crucial. Imaging exams have been a main clinical diagnostic criteria for the 2019 novel coronavirus disease (COVID-19) in China. Imaging features of multiple patchy areas of ground glass opacity and consolidation predominately in the periphery of the lungs are characteristic manifestations on chest CT and extremely helpful in the early detection and diagnosis of this disease, which aids prompt diagnosis and the eventual control of this emerging global health emergency.

\section{Key Points}

- In December 2019, China, an outbreak of pneumonia caused by a novel, highly contagious coronavirus raised grave concerns and posed a huge threat to global public health.

- Among the infected patients, characteristic findings on CT imaging include multiple, patchy, ground-glass opacity, crazypaving pattern, and consolidation shadows, mainly distributed in the peripheral and subpleural areas of both lungs, which are very helpful for the frontline clinicians.

- Imaging examination has become the indispensable means not only in the early detection and diagnosis but also in monitoring the clinical course, evaluating the disease severity, and may be presented as an important warning signal preceding the negative RT-PCR test results.
\end{abstract}

Keywords Coronavirus $\cdot$ Pneumonia $\cdot$ Diagnostic imaging $\cdot$ Multidetector computed tomography $\cdot$ Mass chest X-ray

Electronic supplementary material The online version of this article (https://doi.org/10.1007/s00330-020-06827-4) contains supplementary material, which is available to authorized users.

Shihua Zhao

cjr_zhaoshihua@163.com

$\triangle$ Minjie Lu

coolkan@163.com; minjie.lu@nih.gov

1 Department of Magnetic Resonance Imaging, Fuwai Hospital, National Center for Cardiovascular Diseases, Chinese Academy of Medical Sciences and Peking Union Medical College, \#167 Beilishi Road, Xicheng District, Beijing 100037, China

2 Department of Clinical Medicine, Wuhan University, Wuhan, Hubei 430071, China

3 Department of Radiology, Zhongnan Hospital of Wuhan University, Wuhan, Hubei 430071, China
4 Department of Health and Human Services, National Heart, Lung and Blood Institute (NHLBI), National Institutes of Health (NIH), Bethesda, MD 20892, USA

5 Department of Radiology, Johns Hopkins University School of Medicine, Baltimore, MD 21205, USA

6 Department of Radiology, University of Cambridge, Cambridge, UK

7 Department of Engineering, University of Cambridge, Cambridge CB2 1TN, UK

8 Key Laboratory of Cardiovascular Imaging (Cultivation), Chinese Academy of Medical Sciences, Beijing 100037, China 


\section{Abbreviations}

COVID-19 Coronavirus disease 2019

SARS-CoV-2 Severe acute respiratory syndrome coronavirus 2

\section{Introduction}

In December of 2019, the city of Wuhan, located in the Hubei province of China, became the epicenter of an outbreak of a pneumonia of unknown cause. This pneumonia was later confirmed to be secondary to infection by a novel coronavirus. This virus was originally called the 2019 novel coronavirus (2019-nCoV), but on February 11, 2020, the disease was named COVID-19 by the World Health Organization (WHO), and the virus was named "severe acute respiratory syndrome coronavirus 2" (SARS-CoV-2) by the International Committee on Taxonomy of Viruses (ICTV). It is believed that the COVID-19 outbreak has a zoonotic origin and began in the Huanan wet seafood wholesale market in Wuhan. SARS-CoV-2 has proven to be extremely contagious and spreads easily from person to person. Cases of COVID-19 have now spread beyond Wuhan to many other provinces in China as well as other countries, which has raised international attention and concern. As of March 12, 2020, more than 81,000 human infections with SARS-CoV-2 and at least 3100 deaths related to COVID-19 have been confirmed in China alone. Although the epidemic situation in China has been relatively under control, the situation outside China is getting worse. So far, the COVID-19 viral disease that has swept into at least 114 countries with more than 60,000 cases diagnosed and more than 4000 people killed is now officially a pandemic, as WHO Director-General Tedros Adhanom Ghebreyesus announced in Geneva on March 11.

At first, COVID-19 appeared in a few clusters and was more likely to affect persons with advanced age or other comorbidities, but it is now more widespread. Infection can result in severe pneumonia and even fatal respiratory diseases such as acute respiratory distress syndrome (ARDS) [1, 2]. The main clinical presentation includes fever, dry cough, fatigue, and malaise and/or non-specific upper respiratory tract infection symptoms that may not be particularly noticeable. As previously reported [1], patients with severe illness can develop dyspnea and some even developed ARDS and required ICU admission and oxygen therapy. Laboratory findings of patients infected with SARS-CoV-2 include lymphopenia, elevated CRP, and erythrocyte sedimentation rate. Genetic sequencing of SARS-CoV-2 has enabled the rapid development of point-of-care real-time RT-PCR diagnostic tests specific for COVID-19 [3, 4]. Novel coronavirus nucleic acids are detected in throat swabs, sputum, lower respiratory tract secretions, and blood. At present, the diagnosis of COVID-19 is primarily based on the patient's epidemiological history, clinical symptoms, chest imaging findings, and etiological evidence. Viral nucleic acid testing is an important diagnostic criterion; however, it has some limitations. False negatives from viral nucleic acid testing arise from the uneven quantity of detection technology, disease characteristics (asymptomatic patients), and status of epidemic prevention and control, as well as errors in clinical sampling [5]. COVID-19 is highly contagious; thus, early detection and diagnosis are of paramount importance to isolate suspected cases and contacts to control the outbreak. The challenges in identification of pathogens by viral nucleic acid testing have made some frontline clinicians propose $\mathrm{CT}$ as a diagnostic method for identifying COVID-19 patients. This review aims to briefly introduce the novel coronavirus pneumonia and highlight the value of imaging in its diagnosis.

\section{Etiology and epidemiology}

Coronaviruses typically result in respiratory and enteric infections and affect both animals and humans. Six coronavirus species are known to cause human infection, two of which typically can cause severe respiratory illness and fatalities: SARS-CoV (severe acute respiratory syndrome coronavirus) which had an outbreak in Guangdong, China, between 2002 and 2003 and MERS-CoV (Middle East respiratory syndrome coronavirus) which had an outbreak in 2012 outbreaks in the Middle East [6, 7]. By January 7, 2020, Chinese scientists had isolated the novel coronavirus from patients in Wuhan [3]. It has been reported [8] that SARS-CoV-2 was more similar to two bat-derived coronavirus strains (bat-SL-CoVZC45 and bat-SL-CoVZXC21) than to known human-infecting coronaviruses, including the virus that caused the SARS outbreak of 2003. Molecular modeling has shown structural similarity between the receptor-binding domains of SARS-CoV and SARS-CoV-2, which suggests that SARS-CoV-2 may use angiotensin-converting enzyme 2 (ACE 2) as a receptor. The differences of the etiological characteristics among SARSCOV, MERS-COV, and SARS-CoV-2 are shown in Table 1.

On December 31, 2019, the Health Commission of Hubei province in China announced that 27 patients were afflicted with this mysterious disease, which was subsequently discovered to be COVID-19. The outbreak is believed to zoonotic in origin and likely started at the Huanan wet seafood wholesale market in Wuhan. SARS-CoV-2 is highly contagious and the basic reproductive number of the virus was estimated to be $2 \cdot 2$ [9, 10]. COVID-19 has spread beyond Wuhan to other cities in China and other countries. In recent days, there is a significant growth trend in other countries, especially in South Korea, Japan, Italy, and Iran. The current global situation as of March 13 2020, of COVID-19 is shown in Fig. 1. 
Table 1 Etiological characteristics of SARS-COV, MERS-COV, and SARS-CoV-2

\begin{tabular}{llllll}
\hline Virus & Genus & Intermediate host & Reservoir host & Receptor & Origin \\
\hline SARS-COV-2 & $\beta$-coronavirus & $?$ & Bat & ACE2 & Wuhan, China \\
SARS-COV & $\beta$-coronavirus & Civet & Bat & ACE2 & Guangzhou, China \\
MERS-COV & $\beta$-coronavirus & Dromedary & Bat & CD26/DPP4 & Saudi Arabia \\
\hline
\end{tabular}

In the available reports, the bat is the most possible host of the SARS-CoV-2 and another animal may act as an intermediate host between bats and humans $[8,11]$. To this point, the main infection sources were the patients infected with SARSCoV-2. COVID-19 mainly spread via human-to-human transmission through respiratory droplets and contact. The human population in general is susceptible, while the elderly as well as people with underlying diseases show more serious conditions after infection [12]. Although most people were reported to have a good prognosis of COVID-19, it is unknown whether there are potential impacts on the patients in the future and the sequelae are not available. In the case of the SARS outbreak, significant impact on pulmonary function, functional capacity, and quality of life was found during patient followup [13, 14]. The additional and detailed information about "Etiology and Epidemiology" is provided in Electronic Supplementary Material (ESM).

\section{Imaging features}

According to current diagnostic criteria, identification of the viral pathogen via nucleic acid detection (usually from swab test) is considered as the gold standard and formative assessment for the diagnosis of COVID-19 [12]. However, due to various problems of virus detection in the clinical setting such as a shortage of supply test kits, irregular sampling of samples, laboratory error, insufficient viral material in the specimen, improper extraction of nucleic acid from clinical materials, and contaminatory and technical problems, there have been false negatives. Health care workers on the front line have found the diagnostic value of imaging to be increasingly valuable, which has translated over into clinical diagnosis of COVID-19 patients [12]. In the clinical work of this epidemic, the radiologists play a crucial role in the rapid identification and early diagnosis of a suspected patient - this can be of great benefit not only to the patient but also to the larger public health surveillance and response systems.

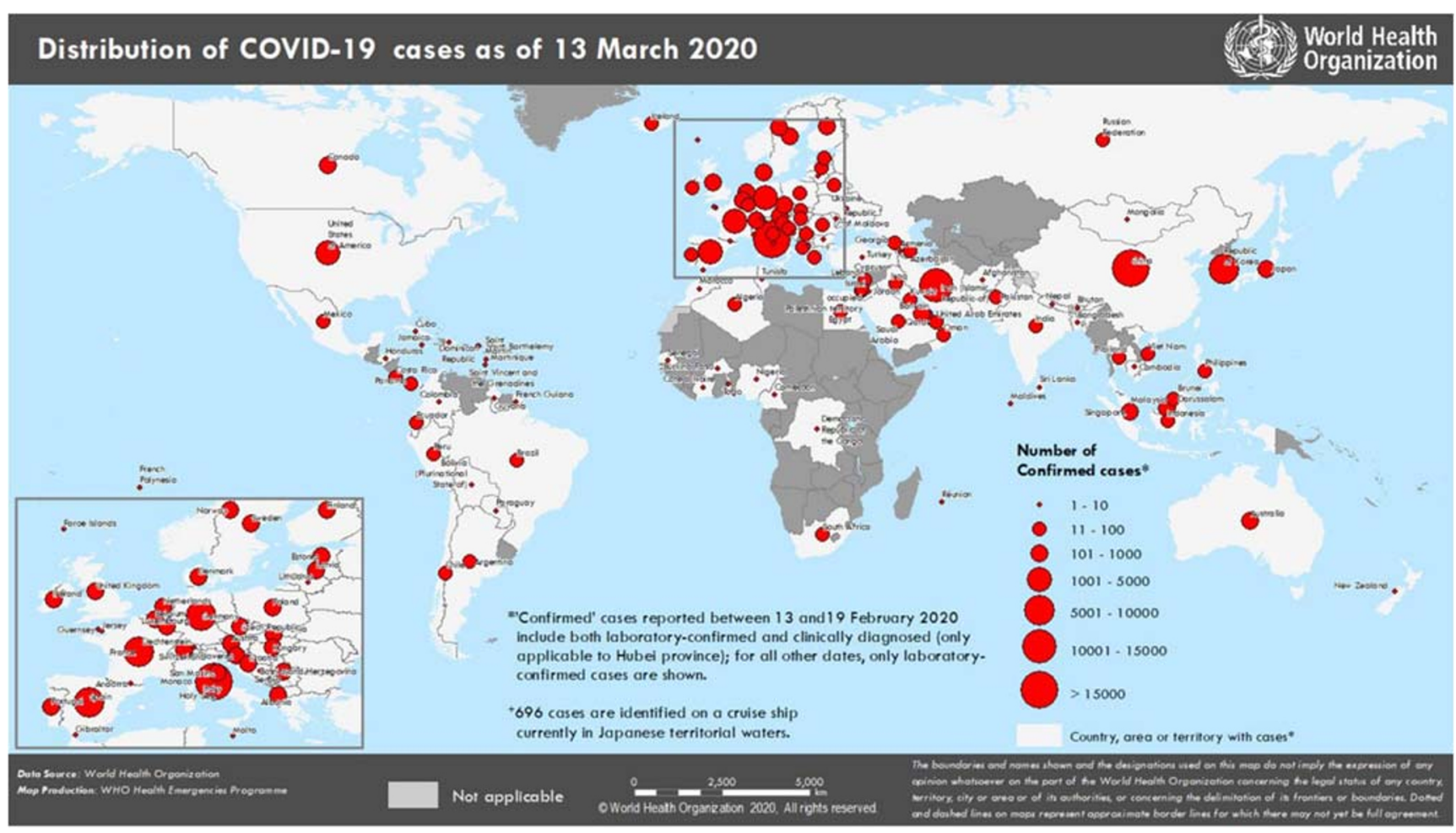

Fig. 1 Countries, territories, and areas with reported confirmed cases of COVID-19, as of March 13, 2020. https://www.who.int/emergencies/diseases/ novel-coronavirus-2019/situation-reports 


\section{Chest radiograph}

Chest radiograph of COVID-19 is not routinely recommended in clinical practice currently because they are insensitive to detecting COVID-19 in the early stage. However, chest radiography may have some utility, with the potential to serve as a screening tool on the frontlines in medical settings with limited resources or in cases where the patient's physical condition does not allow for transport to the radiology department CT scanner. As the disease progresses beyond the early stage, chest radiography can detect multiple patchy opacities throughout the lungs. These opacities eventually become confluent and severe cases may appear as a "whited out lung" [15]. In more advanced cases, in addition to the ground glass opacity and consolidation, even pleural fluid (in a severe case) has been reported on the chest radiographs $[2,16]$. Progressive opacities and consolidation have been observed on chest radiographs over the time course of the illness [17]. In the first case of COVID-19 reported in the USA, there were no obvious abnormalities on the chest radiograph during the early stages after the onset of symptoms. However, on day 9 of the illness, an increased area left basilar opacity was visible on chest radiography. Then, stable streaky opacities in the lung bases were visible, and the opacities have steadily increased over time [18].

\section{Chest computed tomography}

Computed tomography (CT) imaging is strongly recommended because it is very sensitive to detecting early disease, assessing the nature and extent of lesions, and discovering subtle changes that are often not visible on chest radiography. The imaging features of lesions are always described with the following factors: distribution, quantity, shape, pattern, density, and concomitant signs [19].

The typical chest CT imaging characteristics of COVID-19 include multiple, peripheral, bilateral, patchy, sub-segmental, or segmental ground glass opacities and areas of consolidation, which are mostly distributed along the bronchovascular bundles and subpleural space. The presence of associated interlobular septal thickening in the areas of ground glass opacity can give a crazy paving appearance. Air bronchograms with the areas of consolidation and bronchial wall thickening are often present. More rarely, there is a thickening of the adjacent pleura or interlobar pleura, and a small amount of pleural effusion. There is no obvious lymphadenopathy [15] (Tables 2,3). The imaging features mentioned above are consistent with the findings from 81 patients in a recently reported article, in which the predominant pattern of abnormality observed was bilateral, peripheral, ill-defined, and ground glass opacification, mainly involving the right lower lobes [20]. Furthermore, in the currently available reports, the most common chest CT findings in COVID-19 patients are the peripheral areas of ground glass opacity/consolidation (without subpleural sparing) which are bilateral in distribution [21-23]. In one study, of 21 patients, most had more than two lobes affected $(15$ of $21,71 \%$ ) with bilateral involvement (16 of 21, 76\%) [24]. Another study showed that the most common patterns of COVID-19 on thin-section CT images are pure ground glass opacity, ground glass opacity with intra- and/or interlobular septal thickening, and ground glass opacity with consolidation and consolidation, with prominent distribution in the posterior and peripheral part of the lungs [25]. In a large research cohort study, chest CT findings showed a bilateral distribution of patchy ground glass opacity and consolidation in 138 patients [26]. Typical chest radiograph and chest CT of COVID-19 are shown in Figs. 2 and 3.

In "Expert Recommendations from the Chinese Medical Association Radiology Branch," chest CT manifestations of COVID-19 are divided into three stages: early, advanced, and severe, based on the extent of lesion involvement [15]. This current clinical guideline recommends dividing COVID-19 into four stages according to the time of onset and the response of body to the virus with additional dissipation stage (Fig. 4).

1. Early stage: Chest CT shows single or multiple scattered patchy or conglomerate ground glass opacities, predominantly in the middle and lower lungs and along the bronchovascular bundles. These ground glass lesions are often located in peripheral and subpleural areas of the lung. Intra- and interlobular septal thickening sometimes present in the areas of ground glass opacity can give a crazy paving pattern. The pathological process during this stage is dilatation and congestion of the alveolar septal capillary, exudation of fluid in alveolar cavity, and interlobular interstitial edema [19]. One patient had normal chest $\mathrm{CT}$ at initial presentation; however, 3 days later the disease progressed and developed a solitary rounded ground glass lesion in the right lower lobe, indicating this pattern may represent the very first radiologically visible manifestation in patients infected with SARS-CoV-2 [24].

2. Advanced stage: At this stage, chest CTs will show new lesions that are similar to the earlier lesions described above. In addition, findings from the early stage of disease increase in density and extent, coexisting with the new areas of disease. As areas of consolidation grow, air bronchograms are often present in the areas of consolidation. A previous case report described a patient who had evolved into a mixed pattern of ground glass opacities and consolidation by 15 after onset of symptoms [27]. The pathological features in this stage are the accumulation of a cell-rich exudate in the alveolar cavity, vascular expansion, and exudation in the interstitium. The fibrous exudation connects each alveolus through the interalveolar space to form a fusion state [19]. 
Table 2 The typical features on CT imaging of COVID-19

\begin{tabular}{ll}
\hline Parameter & Characteristic manifestations on CT imaging \\
\hline Density & Ground glass opacity and consolidation, possible interlobular septal thickening \\
Shape & Patchy, sub-segmental, or segmental \\
Distribution & Mid and lower lungs along the bronchovascular bundles with bilateral involvement \\
Location & Peripheral and subpleural areas of the lung parenchyma \\
Concomitant signs (variable) & Air bronchogram, a small amount of pleural effusion, no obvious lymphadenopathy \\
\hline
\end{tabular}

3. Severe stage: As the disease further progresses, chest CT shows diffuse consolidation of the lungs of varying density secondary to the fibrous exudate into the alveolar cavity, air bronchograms, and bronchial dilation. Nonconsolidated areas of the lung appear as patchy ground glass opacity. When most of the lungs are involved, the lungs appear as a "whited out lung." The pleura is thickened and there can be a small amount of pleural effusion.

4. Dissipation stage: Images show gradual resolution of the ground glass opacity and consolidation in the lungs with some residual curvilinear opacities compatible with fibrosis.

After isolation and treatment, a majority of patients infected by COVID-19 stabilize and gradually recover and on imaging the areas of disease decrease correspondingly. However, in a small number of patients with underlying disease or advanced age, the disease progresses during the treatment with the scope of the lesions in the lungs expanding and increasing density, eventually appearing as "whited out lungs."

Table 3 Frequency of chest CT findings in COVID-19

\begin{tabular}{lll}
\hline CT signs & Frequency & Stage \\
\hline Ground glass opacity & ++++ & E/A/S \\
Consolidation without ground glass opacity & ++ & $\mathrm{S}$ \\
Ground glass opacity and crazy paving & ++ & $\mathrm{E} / \mathrm{A} / \mathrm{S}$ \\
Ground glass opacity with consolidation & +++ & $\mathrm{E} / \mathrm{A} / \mathrm{S}$ \\
Patchy ground glass opacity & +++ & $\mathrm{E}$ \\
Bilateral distribution & ++++ & $\mathrm{E} / \mathrm{A} / \mathrm{S} / \mathrm{D}$ \\
Peripheral distribution & +++ & $\mathrm{E}$ \\
Air bronchogram & ++ & $\mathrm{E} / \mathrm{A} / \mathrm{S}$ \\
Pleural effusion & + & $\mathrm{S}$ \\
Strip-like opacity & + & $\mathrm{D}$ \\
\hline
\end{tabular}

The appearance frequency of each CT characteristic is described in order from low to high as $(+\sim++++) ; E, A, S$, and $D$ stand for stage early, advanced, severe, and dissipation

\section{Role of imaging in COVID-19}

On the frontlines of fighting the COVID-19 epidemic, chest imaging (particularly chest CT) now has an irreplaceable role in the early diagnosis of COVID-19 as well as monitoring the disease's clinical course. COVID-19 has characteristic manifestations in the lung that are readily detected on chest CT as described in some recent publications [15, 24, 25]. In several prior reports, chest CT showed progression of disease that corresponded with worsening clinical symptoms and also disease resolution as the patients clinically recovered $[21,22$, 27]. In a recent report [20], the predominant pattern of abnormality in COVID-19 observed on the basis of the interval between symptom onset and the first CT scan has some differences. Lesions quickly evolved from focal unilateral to diffuse bilateral ground glass opacities that progressed to or coexisted with consolidations within 1-3 weeks, indicating the change through clinical course which was also reflected on the number of involved lung segments. In a prior study of 51 patients, the absolute number of lung findings increased with the time from symptom onset and lesions with consolidations including ground glass opacities with consolidation and pure consolidation showed mildly positive correlation with the time between symptom onset and the CT [25]. Another report demonstrated mild or moderate progression of disease as manifested by increasing extent and density of lung opacities during follow-up [24]. In the context of typical clinical presentation and exposure history, some patients may present with negative results of RT-PCR for COVID-19 but have characteristic imaging features of COVID-19 on chest CT. Subsequently, these patients whose labs were initially negative for COVID-19 did test positive later with repeat swab tests. Thus, in patients at high risk for COVID-19, chest CT evidence of viral pneumonia may precede negative RT-PCR test results and present as an important warning signal. A combination of repeated swab tests and CT scanning may be helpful for individuals with high clinical suspicion of COVID19 but negative RT-PCR screening $[28,29]$. Chest CT has a high sensitivity for diagnosis of COVID-19. In a report of 51 patients with chest CT and RT-PCR assay performed within 3 days, the sensitivity of chest CT was greater than that of RTPCR for COVID-19 at initial patient presentation (98\% vs $71 \%$, respectively, $p<0.001$ ) [30]. In another study of 1014 
Fig. 2 Chest radiograph (a) in a 61-year-old man shows bilateral patchy, somewhat nodular opacities in the mid to lower lungs [16]. Unenhanced computed tomography (CT) images (b) in a 33-year-old woman., Images show multiple ground glass opacities in the periphery of the bilateral lungs. The bilateral, peripheral patterns of opacities without subpleural sparing are common and characteristic CT findings of the 2019 novel coronavirus pneumonia [22]. Chest CT image of a 71-year-old male (c) shows consolidation in the peripheral right upper lobe and a patchy area of ground glass opacity with some associated consolidation intra- and interlobular septal thickening within the left upper lobe [25]

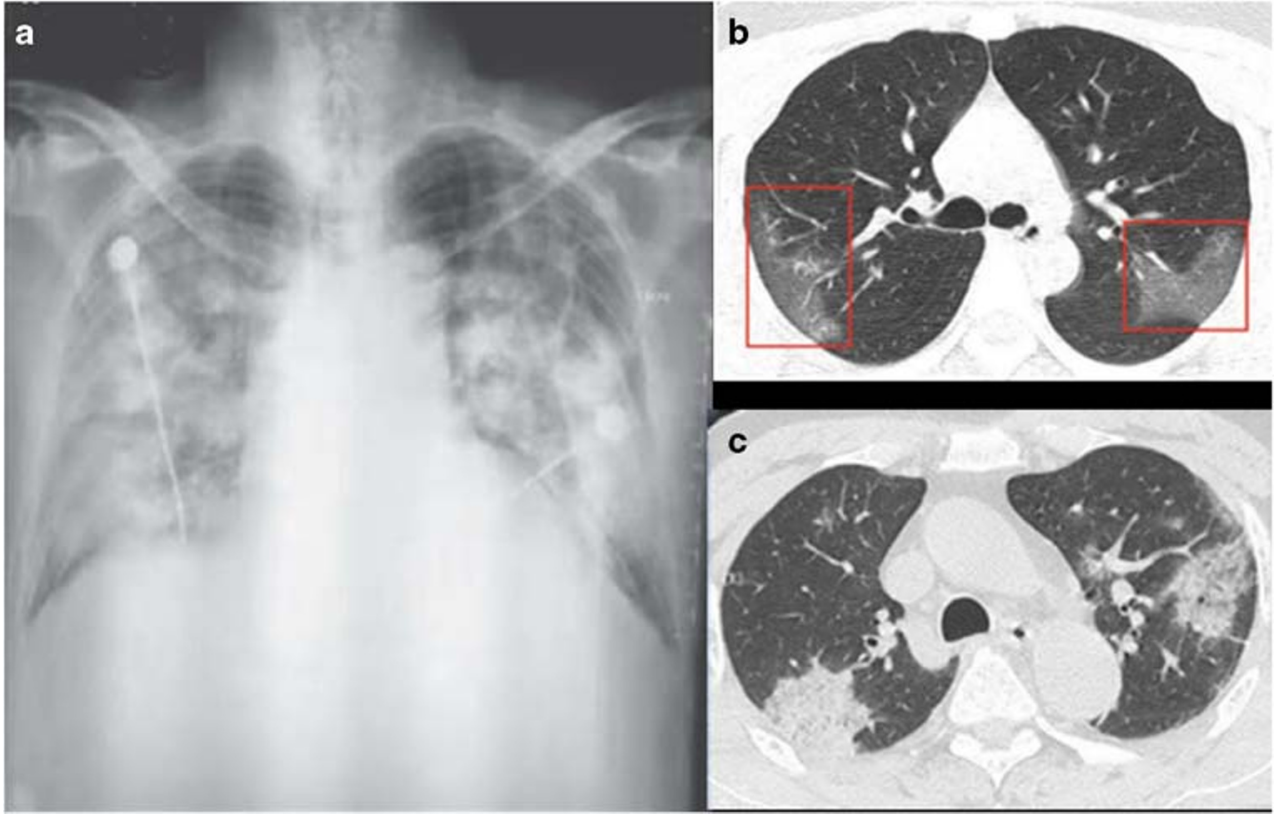

patients, 59\% had positive RT-PCR results, and $88 \%$ had positive chest CT scans. The sensitivity of chest CT in suggesting COVID-19 was $97 \%$ based on positive RT-PCR results. Between $60 \%$ and $93 \%$ of cases had initial positive CT consistent with COVID-19 prior (or parallel) to the initial positive RT-PCR results [31]. In addition, chest CT can evaluate disease severity. In terms of the percentage of pneumonia lesions in the entire lung volume, the difference between the earlier stage patients and the severe stage patients is statistically significant [32]. In an initial prospective analysis of the clinical features of 41 patients, chest CT images of ICU patients on admission showed bilateral multiple lobular and subsegmental areas of consolidation, and overall had more imaging abnormalities than non-ICU patients [1]. Older patients may have more systemic symptoms, more extensive lung involvement, and worse prognosis than younger patients [33]. Thus, the typical CT findings of COVID-19 can not only help early screening suspected cases, but can also monitor the clinical progression and may predict severe complications such as acute respiratory diseases. With high sensitivity for COVID19 , chest CT would play a very important role in the diagnosis and treatment of COVID-19 in the high-risk regions and
Fig. 3 Typical CT findings of COVID-19. Chest CT (a) in a 75 year-old male show multiple patchy areas of pure ground glass opacity (GGO) and GGO with reticular and/or interlobular septal thickening [25]. Chest CT image of a 38-year-old male (b) shows multiple patches, grid-like lobule, and thickening of interlobular septa, typical "paving stone-like" signs [19]. An axial CT image obtained in 65-year-old female (c) shows bilateral ground glass and consolidative opacities with a striking peripheral distribution [23]. CT image of a 65 -year-old male (d) shows large consolidation in the right middle lobe, patchy consolidation in the posterior and basal segment of right lower lobe, with air bronchogram inside [19]

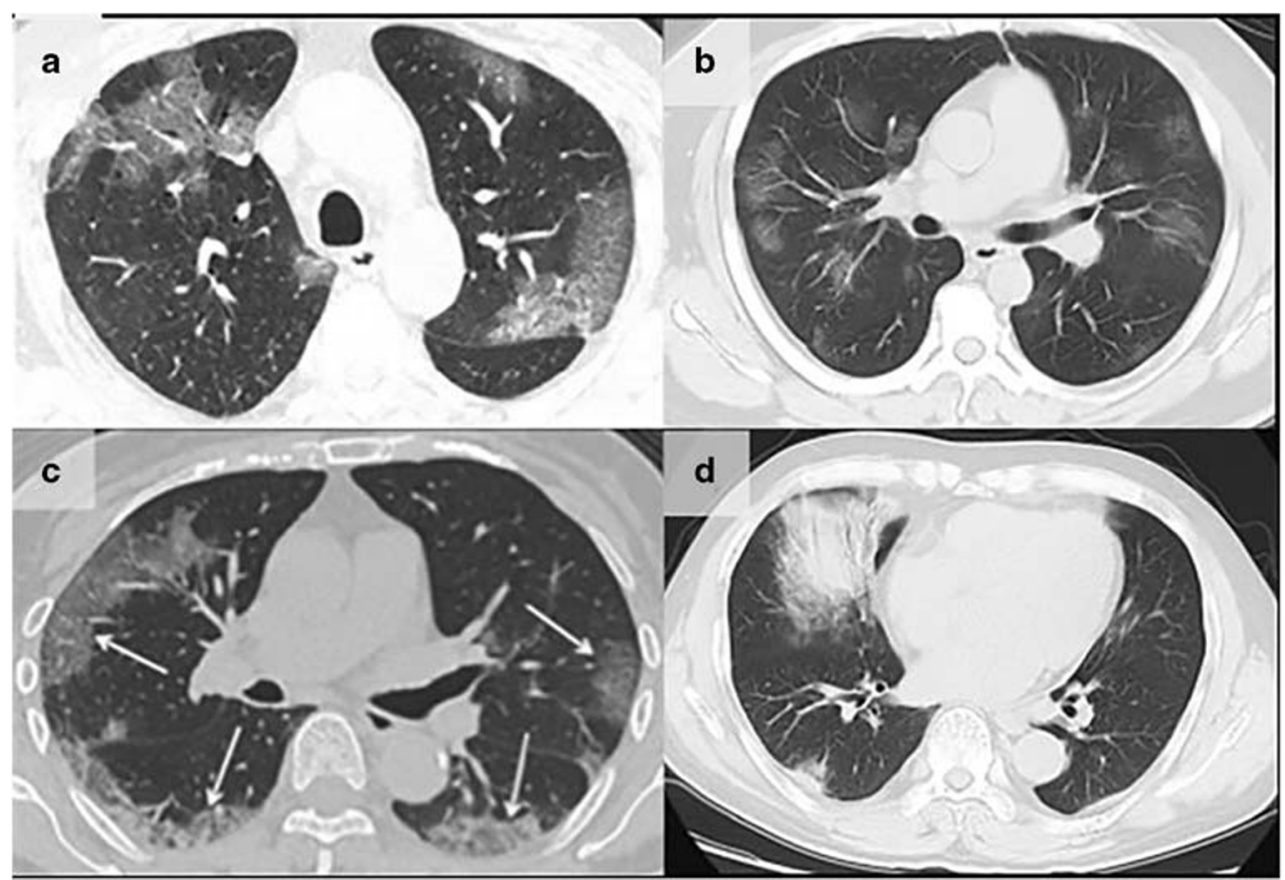




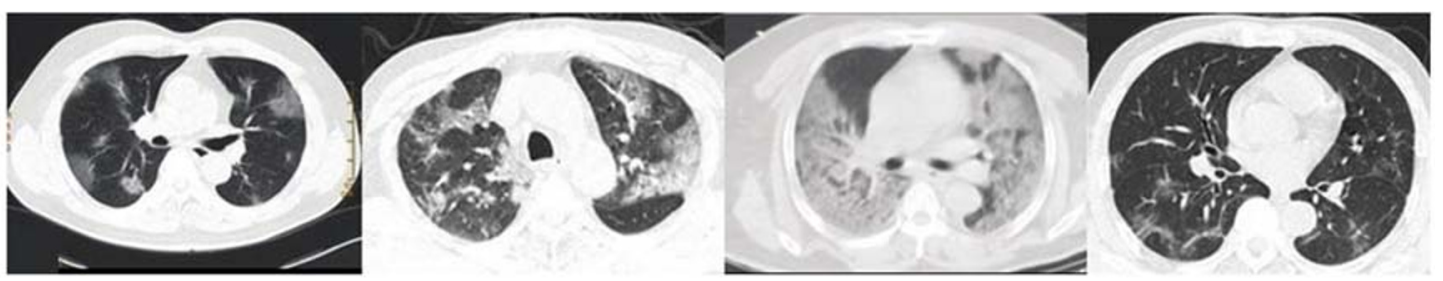

\section{Early stage}

Single or multiple scattered patchy ground-glass opacities, predominately distributed in the peripheral and subpleural area of the lung. A crazy-paving pattern, secondary to intralobular and interlobular septal thickening can be seen in this stage

\section{Advanced stage}

Increased extent and density of bilateral lung parenchymal opacities. In this image, there are both areas of ground glass opacification and areas of consolidation in both lungs, which coexist and have varying sizes and presence of airbronchogram.
Severe stage

Diffuse consolidation of the lung parechyma with uneven density, air bronchi and bronchial dilation, which may be present as "whited out lung"on a corresponding chest radiograph

\section{Dissipation stage}

Areas of ground glass opacity and consolidation have nearly completely resolved, leaving some residual curvilinear areas of density.

Fig. 4 CT manifestations of different stages of COVID-19

countries. In some extreme situations in very high-risk areas (Wuhan, China), CT was the one of main diagnostic criteria for this disease due to the relatively high false negative of RTPCR. For some low-risk regions and countries, positive predictive value of CT alone or adding CT to RT-PCR should be adjusted. The proposition of CT could be tempered to some degree. Therefore, to be more accurate, the role of chest CT in COVID-19 should be assessed based on the prevalence of the disease in each area.

\section{Differential diagnosis}

The CT appearance of COVID-19 shares some similarities with other diseases that cause viral pneumonia, including influenza viruses, parainfluenza virus, adenovirus, respiratory syncytial virus, rhinovirus, human metapneumovirus, etc. (Table 4). In particular, those within the same viridae (SARS and MERS) have great similarities in imaging findings because they belong to the same coronaviridae family, and thus need to be excluded through clinical manifestations and laboratory pathogen detection. Imaging in MERS pneumonia can also show ground glass lesions in the subpleural and basal parts with consolidation, and fibrosis changes can be left after healing [34]. In the pneumonia patients infected by respiratory syncytial virus, chest CT mainly manifested as small centrilobular nodules and areas of consolidation which are often asymmetrically distributed in the lungs. Adenovirus pneumonia shows bilateral multifocal ground glass opacities with patchy consolidations on CT images and may show lobar or segmental distribution. Human parainfluenza virus pneumonia may show centrilobular nodules with bronchial wall thickening that differentiates it from the imaging appearances of the other viruses. Radiographs in patients with influenza pneumonia show bilateral patchy areas of ground glass opacity with or without focal areas of consolidation, usually in the lower lobes [34]. In a report on H1N1 influenza infection, in addition to the most common findings of ground glass opacity, interlobular septal thickening, and centrilobular nodules were the second most frequent findings [35]. Rapidly progressive ground glass opacities and consolidations with air bronchograms and interlobular septal thickening, with right

Table 4 Differential diagnosis of different viral pneumonia

\begin{tabular}{ll}
\hline Virus & Imaging characteristics \\
\hline MERS-COV & $\begin{array}{c}\text { Ground glass lesions in the subpleural and basal portions of the lung parenchyma with areas of consolidation; } \\
\text { fibrotic changes can be present after healing }\end{array}$ \\
H1N1 & $\begin{array}{l}\text { Ground glass opacity, interlobular septal thickening, and centrilobular nodules } \\
\text { H7N9 }\end{array}$ \\
$\begin{array}{l}\text { Guman parainfluenza virus } \\
\text { Respiratory syncytial virus }\end{array}$ & $\begin{array}{l}\text { Centrilobular nodules with bronchial wall thickening, findings which differentiate it from other viral infections } \\
\text { Adenovirus pneumonia }\end{array}$ \\
\hline
\end{tabular}


lower lobe predominance, are the main imaging findings in H7N9 pneumonia [36]. Viruses are a common cause of respiratory infection and recognition of viral pneumonia patterns may help in the differentiation among viral pathogens, while the definite diagnosis is achieved by laboratory detection of virus.

In addition, COVID-19 also needs to be distinguished from mycoplasma pneumonia, chlamydia pneumonia, and bacterial pneumonia. Other diseases that need to be identified are vasculitis, acute interstitial pneumonia, connective tissue-related lung disease, and cryptogenic organizing pneumonia [15].

\section{Conclusions}

The COVID-19 initially began in China; however, it is extremely contagious and has spread beyond China to many other countries, raising concerns and posing not only a huge threat to global public health but also a huge economic burden and panic to society. Early disease recognition can prompt early patient isolation and early diagnosis and treatment. In the current situation, imaging of COVID-19, particularly with chest CT, has a very high value because it shows characteristic manifestations and has enabled frontline clinicians to have primary diagnosis in their first contact with suspected patients, even in the presence of initially false negative lab results. Earlier diagnosis with the aid of imaging allows for early containment and response to this communicable disease as well as overcoming the outbreak as soon as possible through a joint effort.

Acknowledgments We are very grateful to the worldwide front-line medical staff for their dedication in the fighting against the outbreak of COVID-19, despite the potential threat to their own lives and the lives of their families. And we also believe this pandemic will be controlled by the efforts of all mankind.

Funding information This study was funded by Construction of Key Laboratory (Cultivation) of Chinese Academy of Medical Sciences(2019PT310025), National Foreign Expert Talent Project (G20190001630), Education Reform Project of Peking Union Medical College (10023201900204), and Clinical and Translational Fund of Chinese Academy of Medical Sciences (2019XK320063).

\section{Compliance with ethical standards}

Guarantor The scientific guarantor of this publication is Minjie Lu.

Conflict of interest The authors have no conflict of interest to disclose.

Statistics and biometry No complex statistical methods were necessary for this paper.

Informed consent Written informed consent was not required for this study because this is a review of literature.
Ethical approval Institutional Review Board approval was not required because this is a review of literature.

Methodology

- N/A

\section{References}

1. Huang C, Wang Y, Li X et al (2020) Clinical features of patients infected with 2019 novel coronavirus in Wuhan, China. Lancet 395(10223):497-506

2. Chen N, Zhou M, Dong X et al (2020) Epidemiological and clinical characteristics of 99 cases of 2019 novel coronavirus pneumonia in Wuhan, China: a descriptive study. Lancet 395(10223):507-513

3. Wang C, Horby PW, Hayden FG, Gao GF (2020) A novel coronavirus outbreak of global health concern. Lancet 395(10223):470 473

4. Yu F, Du L, Ojcius DM, Pan C, Jiang S (2020) Measures for diagnosing and treating infections by a novel coronavirus responsible for a pneumonia outbreak originating in Wuhan, China. Microbes Infect. https://doi.org/10.1016/j.micinf.2020.01.003

5. Mo Q, Qin W, Fei QH et al (2020) Correctly understand the influencing factors of nucleic acid detection of novel coronavirus. Chin J Lab Med 43(00):E002-E002

6. Ksiazek TG, Erdman D, Goldsmith CS et al (2003) A novel coronavirus associated with severe acute respiratory syndrome. $\mathrm{N}$ Engl J Med 348(20):1953-1966

7. Zaki AM, van Boheemen S, Bestebroer TM, Osterhaus AD, Fouchier RA (2012) Isolation of a novel coronavirus from a man with pneumonia in Saudi Arabia. N Engl J Med 367(19):18141820

8. Lu R, Zhao X, Li J et al (2020) Genomic characterisation and epidemiology of 2019 novel coronavirus: implications for virus origins and receptor binding. Lancet. https://doi.org/10.1016/ s0140-6736(20)30251-8

9. Li Q, Guan X, Wu P et al (2020) Early transmission dynamics in Wuhan, China, of novel coronavirus-infected pneumonia. N Engl J Med. https://doi.org/10.1056/NEJMoa2001316

10. Riou J, Althaus CL (2020) Pattern of early human-to-human transmission of Wuhan 2019 novel coronavirus (2019-nCoV), December 2019 to January 2020. Euro Surveill 25(4)

11. Zhou P, Yang XL, Wang XG et al (2020) A pneumonia outbreak associated with a new coronavirus of probable bat origin. Nature. https://doi.org/10.1038/s41586-020-2012-7

12. General Office of National Health Committee (2020) Office of State Administration of Traditional Chinese Medicine. Notice on the issuance of a programme for the diagnosis and treatment of novel coronavirus (2019-nCoV) infected pneumonia (Trial Version 5)

13. Hui DS, Joynt GM, Wong KT, Gomersall CD et al (2005) Impact of severe acute respiratory syndrome (SARS) on pulmonary function, functional capacity and quality of life in a cohort of survivors. Thorax 60(5):401-409

14. Hui DS, Wong KT, Antonio GE, Tong M, Chan DP, Sung J (2009) Long-term sequelae of SARS: physical, neuropsychiatric, and quality-of-life assessment. Hong Kong Med J 15(Suppl 8)

15. Chinese Medical Association Radiology Branch (2020) Radiological diagnosis of new coronavirus pneumonia: expert recommendations from the Chinese Medical Association Radiology Branch (first edition). Chin J Radiol 54(00):E001-E001

16. Zhu N, Zhang DY, Wang WL et al (2020) A novel coronavirus from patients with pneumonia in China, 2019. N Engl J Med. https://doi. org/10.1056/NEJMoa2001017 
17. Phan LT, Nguyen TV, Luong QC et al (2020) Importation and human-to-human transmission of a novel coronavirus in Vietnam. N Engl J Med. https://doi.org/10.1056/NEJMc2001272

18. Holshue ML, DeBolt C, Lindquist S et al (2020) First case of 2019 novel coronavirus in the United States. N Engl J Med. https://doi. org/10.1056/NEJMoa2001191

19. Jin YH, Cai L, Cheng ZS et al (2020) A rapid advice guideline for the diagnosis and treatment of 2019 novel coronavirus (2019$\mathrm{nCoV}$ ) infected pneumonia (standard version). Mil Med Res 7(1):4

20. Shi H, Han X, Jiang N et al (2020) Radiological findings from 81 patients with COVID-19 pneumonia in Wuhan, China: a descriptive study. Lancet Infect Dis. https://doi.org/10.1016/s14733099(20)30086-4

21. Duan YN, Qin J (2020) Pre- and posttreatment chest CT findings: 2019 novel coronavirus (2019-nCoV) pneumonia. Radiology. https://doi.org/10.1148/radiol.2020200323

22. Lei J, Li J, Qi X (2020) CT imaging of the 2019 novel coronavirus (2019-nCoV) pneumonia. Radiology. https://doi.org/10.1148/ radiol.2020200236

23. Bernheim A, Mei X, Huang MQ et al (2020) Chest CT findings in coronavirus disease-19 (COVID-19): relationship to duration of infection. Radiology. https://doi.org/10.1148/radiol.2020200463

24. Chung M, Bernheim A, Mei X et al (2020) CT imaging features of 2019 novel coronavirus (2019-nCoV). Radiology. https://doi.org/ 10.1148/radiol.2020200230

25. Song FX, Shi NN, Shan F, Zhang ZY, Shen J et al (2020) Emerging coronavirus 2019-nCoV pneumonia. Radiology. https://doi.org/10. 1148/radiol.2020200274

26. Wang D, Hu B, Hu C et al (2020) Clinical characteristics of 138 hospitalized patients with 2019 novel coronavirus-infected pneumonia in Wuhan, China. JAMA. https://doi.org/10.1001/jama. 2020.1585

27. Shi H, Han X, Zheng C (2020) Evolution of CT manifestations in a patient recovered from 2019 novel coronavirus (2019-nCoV) pneumonia in Wuhan, China. Radiology. https://doi.org/10.1148/radiol. 2020200269
28. Xie X, Zhong Z, Zhao W, Zheng C, Wang F, Liu J (2020) Chest CT for typical 2019-nCoV pneumonia: relationship to negative RTPCR testing. Radiology. https://doi.org/10.1148/radiol.2020200343

29. Huang P, Liu T, Huang L et al (2020) Use of chest CT in combination with negative RT-PCR assay for the 2019 novel coronavirus but high clinical suspicion. Radiology. https://oi.org/10.1148/ radiol.2020200330

30. Fang Y, Zhang HQ, Xie J et al (2020) Sensitivity of chest CT for COVID-19: comparison to RT-PCR. Radiology. https://doi.org/10. 1148/radiol.2020200432

31. Ai T, Yang Z, Hou HY et al (2020) Correlation of chest CT and RTPCR testing in coronavirus disease 2019 (COVID-19) in China: a report of 1014 cases. Radiology. https://doi.org/10.1148/radiol. 2020200642

32. Huang L, Han R, Yu PX et al (2020) Correlation between CT and clinical manifestations of different clinical types of new coronavirus pneumonia. Chin J Radiol 54(00):E003-E003

33. Chan JF-W, Yuan S, Kok K-H et al (2020) A familial cluster of pneumonia associated with the 2019 novel coronavirus indicating person-to-person transmission: a study of a family cluster. Lancet 395(10223):514-523

34. Koo HJ, Lim S, Choe J, Choi SH, Sung H, Do KH (2018) Radiographic and CT features of viral pneumonia. Radiographics 38(3):719-739

35. Yuan Y, Tao XF, Shi YX, Liu SY, Chen JQ (2012) Initial HRCT findings of novel influenza A (H1N1) infection. Influenza Other Respi Viruses 6(6):e114-e119

36. Wang QL, Zhang ZY, Shi YX, Jiang YB (2013) Emerging H7N9 influenza A (novel reassortant avian-origin) pneumonia: radiologic findings. Radiology 268(3):882-889

Publisher's note Springer Nature remains neutral with regard to jurisdictional claims in published maps and institutional affiliations. 